\title{
The Design and Evaluation of Easy Care Clothing for Disabled
}

\author{
Shurong $\mathrm{HU}^{1, \mathrm{a}}$, Xiaoxia $\mathrm{Wu}^{2, \mathrm{~b}}$ \\ ${ }^{1}$ Fashion Institute, Zhejiang University of Science and Technology, Hangzhou, 310023, China \\ ${ }^{2}$ Fashion College, Zhejiang Sci-Tech University, Hangzhou, 310008, China \\ a rongerdavy@126.com, b107016@zust.edu.cn
}

Keywords: special requirement for disabled, design of easy care clothing, motion analysis, functional evaluation.

Abstract In this paper, the special requirement for the lower limb disabled was investigated by the interview with doctors, nurses and disabled in rehabilitation center. Based on the requirements, the new easy care clothing for wheelchair users is designed by the functional fabrics used, the fastener selected, and the clothing structure adjusted. Then the easy care clothing is evaluated by motion analysis comparing with the ordinary clothing. The result shows that the convenience of new clothing is obviously higher than ordinary clothing.

\section{Introduction}

China is a country with numerous disabled, and the population with physical disabilities is about 24.72 million. In the recent years, with the improvement of civilization, caring about the "disabled persons", safeguarding the vital interests of them become a hot social concern.

The daily life of the disabled was lack of nurses and inadequate protection of the caring ${ }^{[1]}$. The easy care garments designed for the disabled people especially physical handicapped was need ${ }^{[2]}$. However, in the existing garment market, there are fewer garments designed for the needs of the physically handicapped, and the functional and daily needs are rarely met with the practical needs of the physically handicapped in terms of easy care and comfort ${ }^{[3]}$.

This paper based on the shortage of clothing for the handicapped, the demand for physically handicapped was investigated, and then the easy care clothing was designed for lower limb disabled. The subjective and objective experiments were carried out to evaluate the overall wearing comfort and easy to put on and take off for the new clothing.

\section{The Design of Clothing}

According to the investigate results of demand for lower limb handicapped from the special rehabilitation center, it is known that the lower limb disabilities were easy to produce bed sores and pressure sores because of long sedentary and lying, and the movement rang of them was much greater than health people during dressing and using the toilet, the requirement of the softness and flexibility of fabrics is higher. So the knitted fabrics are chosen for the new clothing.

For the wheelchair users who can self-care basically, because they must turn the wheelchair frequently, so the sleeves need to use high wearing resistant fabrics, and the back and shoulder need to use high elastic fabrics. The crotch and hip should be used antibacterial fabric to decrease the possibility of pressure sores and purulent infection. For the wheelchair users who cannot take care of themselves, because of their inconvenient mobility and lying in bed long time, the body especially the back, crotch, hip are easily to cause bedsore due to bacterial infection, so these parts of clothing should be considered the performance of antibacterial and air permeability.

In order to adapt the daily demand, the easy care clothing designed for disabled is improved different from the ordinary clothing. The main sizes of the clothing are shown in Table1. 
Table 1 The main size of clothing

\begin{tabular}{cccccc}
\hline Clothing & Clothing length & Bust width & Shoulder width & Sleeve length & Cuff width \\
\hline Ordinary (cm) & 65 & 104 & 39.5 & 56 & 25 \\
Easy care (cm) & 70 & 104 & 44 & 56 & 25 \\
\hline Clothing & Pantss length & Upper crotch & Waist width & Hip width & Thigh width \\
\hline Ordinary (cm) & 100.5 & 25 & 74 & 104 & 54 \\
Easy care (cm) & 104.5 & 31.5 & 76 & 104 & 61.5 \\
\hline
\end{tabular}

For the basic self-care wheelchair users, the upper body can move freely, so the placket setting is the same as ordinary clothing, but the magnetic buckle is used to replace plastic button (Fig.1). Because of their lower body disability, the opening of pants which is $16 \mathrm{~cm}$ below from the waist is set in the both sides, and the waist is fastened with velcro (Fig.2). A $27 \mathrm{~cm}$ length special opening is setting in the hip part of pants to easier care.

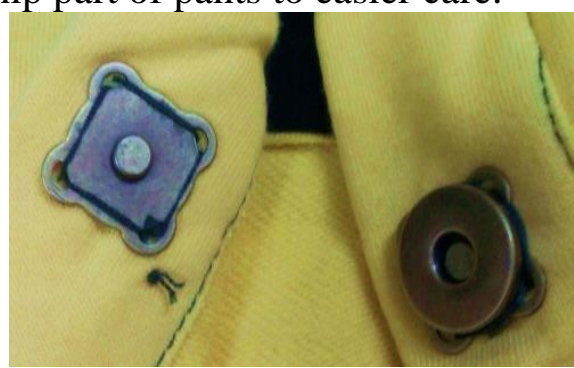

Fig.1 Magnetic buckles using in opening

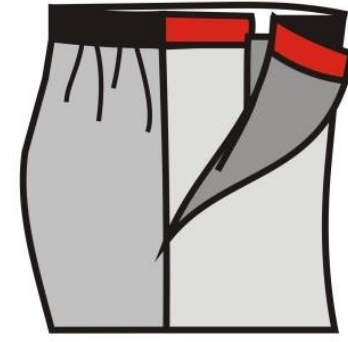

Fig.2 The velcro using in the waist

The $100 \%$ cotton fabric is used in the main parts of body and sleeve, the side of body and the back is using the high elastic Roman, the polyester \& cotton blended antibacterial fabric was used in hip and crotch. The wearable fabric was used in underarm and elbow. The sketch of the new easy care clothing is shown in Fig.3.

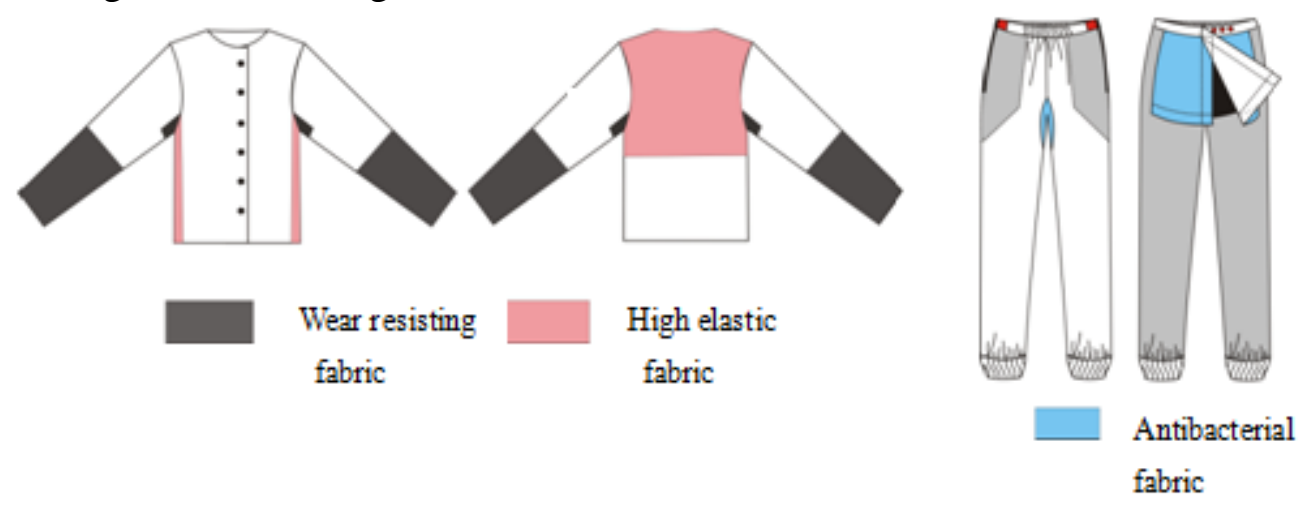

Fig.3 The design of easy care clothing

\section{The Evaluation of Easy Care Clothing}

Six subjects was invited, and they all sit in a chair to simulate the basic self-care wheelchair users to wear ordinary clothing and wheelchair self-care clothing, the camera is used to record during the whole process, and then analyzed frame-by-frame.

\section{Results and Discussion}

The wearing motion analysis for ordinary and new designed clothing of wheelchair user is shown in Fig.2. According to the motion analysis of putting on shirt, we can know that the wearing process and moving range is similar between ordinary and the new designed easy care shirt. 


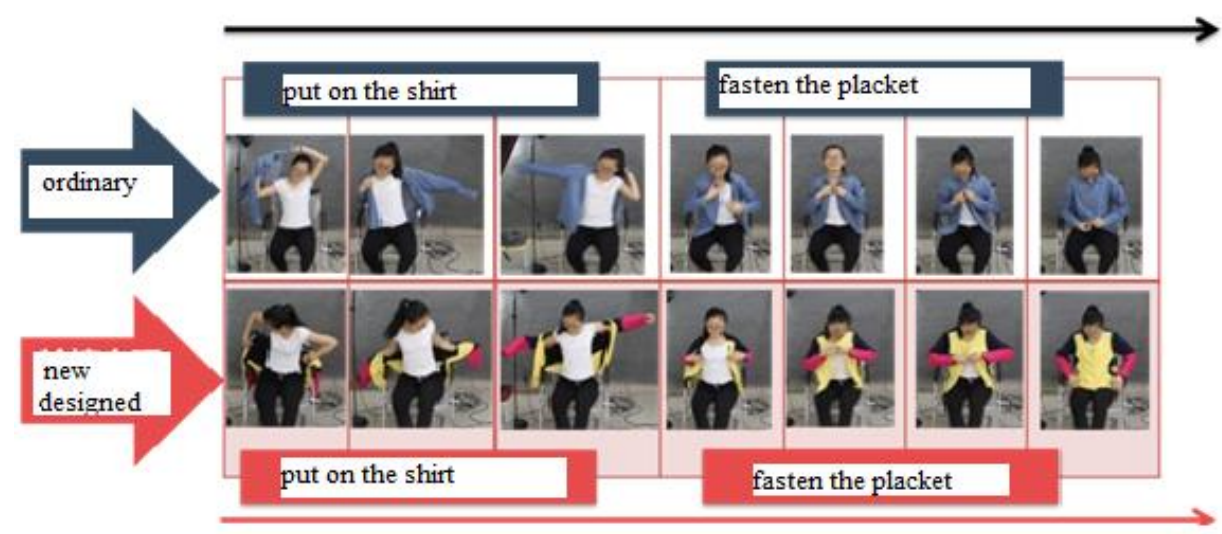

Fig. 2 The motion analysis for putting on shirt of wheelchair user

The step of taking off shirt of ordinary and new designed clothing of wheelchair user is shown in Fig.3.

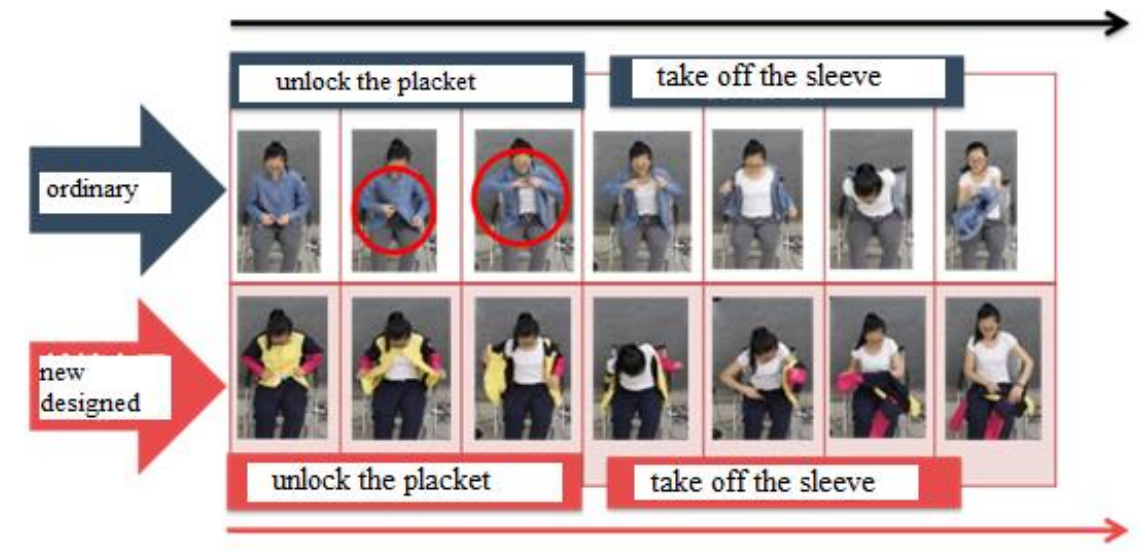

Fig.3 The motion analysis of taking off shirt for wheelchair user

The subjects take off the ordinary shirt need to unlock the six buttons in the center front one by one, the force points for unlocking is the 6 buttons and the corresponding buttonhole, but the new designed easy care shirt can be unlocked just need to pull the side of front bodice, the unlock motion is simplified obviously.

The self-care wheelchair users wear ordinary pants and easy care pants is shown in Fig.4.

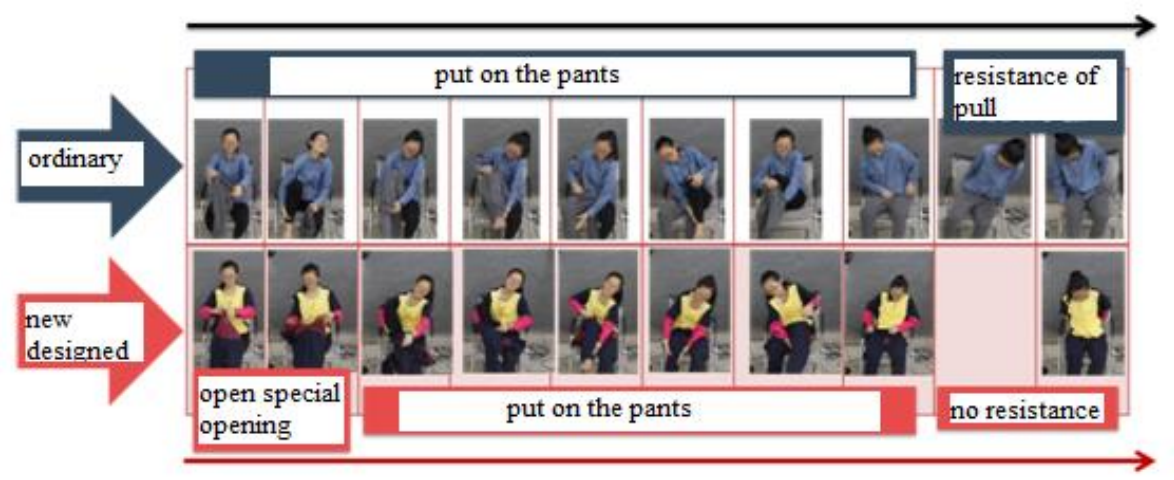

Fig.4 The motion analysis for putting on pants of wheelchair user

When wearing ordinary pants especially on the waist, the subjects need to support the chair with one hand, and pull pants with another hand. Conversely, when wearing the easy care pants, after opening the piece of hip and unlocking the velcro on waist, the motion of support on the chair is not needed, and the motion range in the following wearing motion is reduced obviously. The ordinary pants take more time and energy than the easy care pants. 


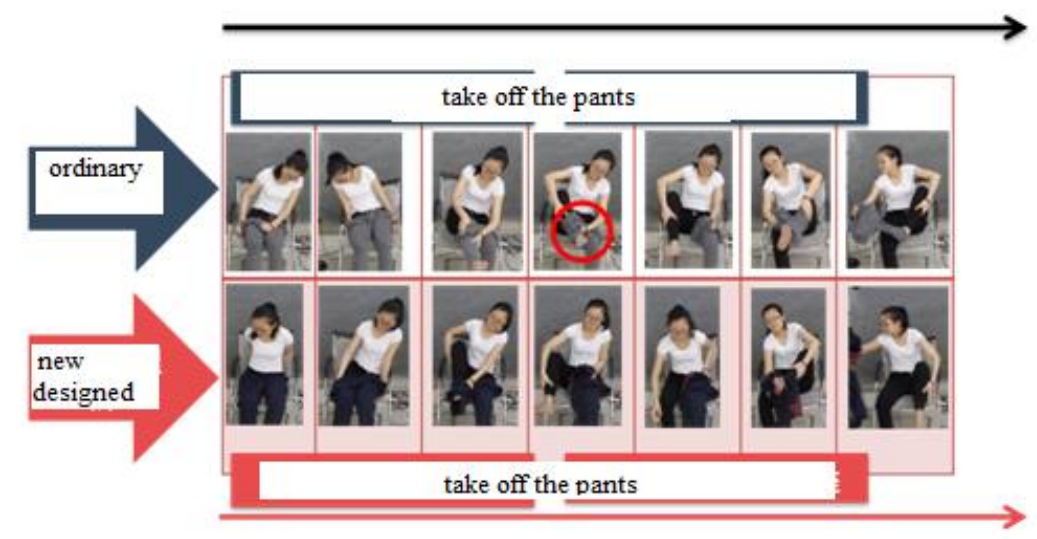

Fig.5 The motion analysis for taking off pants

The difference between taking off the ordinary pants and easy care pants is shown in Fig.5. Because of the limitations of the waist width, it requires a lot of pulling and more time and energy when taking off the ordinary pants. On the other hand, with the using velcro and design of special opening on the waist and hip, the easy care pants has enough width for subjects to take off pants easily. The following motion for taking off is also simplified.

\section{Conclusions}

The different performance fabrics are used in the different zone of the new designed shirt, they can meet the demand of the wheelchair users, and the new shirt gets more function than the ordinary.

The process of putting on is similar between ordinary and the new designed easy care shirt. But the process of taking off is obviously simplified as the special design in the placket.

As the inconvenient of lower limb, the wheelchair user lack of the force support, it causes more time and energy when putting on and taking off the ordinary pants. The new easy care pants with special opening design in the back hip; the difficult of putting on is solved effectively. At the same time, the velcro using in the waist side opening design enhances the waist circumference, the difficult of taking off is weakened obviously.

\section{Acknowledgement}

This work was financially supported by the social sciences union of Zhejiang province (2012N195) and the science and technology planning project of Zhejiang province (2013C31142) and the Zhejiang University of Science and Technology foundation.

\section{References}

[1] S. Van Buuren, S. Eyres, A. Tennant and M. Hopman: Eur J Public Health. Vol.13(2003), p.11-19

[2] N. Nevala, J. Holopainen, O. Kinnunen and O. H.anninen: Appl Ergon.Vol.34(2003), p.557-563

[3] E. M. Badley: Soc Sci Med. Vol.66 (2008), p.23-35 